\title{
Erratum: Nonlinear spectroscopy of trapped ions [Phys. Rev. A 90, 023603 (2014)]
}

\author{
Frank Schlawin, Manuel Gessner, Shaul Mukamel, and Andreas Buchleitner
}

(Received 9 September 2015; published 24 September 2015)

DOI: 10.1103/PhysRevA.92.039903

PACS number(s): 03.75.Hh, 78.47.jh, 37.10.Ty, 78.47.da, 99.10.Cd

Step (iii) of the three-step process to create a motional coherence, the incoherent repumping process of the electronic state [also depicted in Fig. 4(c)], is not suitable since it destroys the motional coherence in Eq. (31). Instead, the desired selective excitation or deexcitation of the motional degree of freedom can be achieved via a coherent redistribution of the electronic excitation into other sublevels of long-lived excited state manifold(s). Step (iii) can thus be replaced by pulses which transfer the $|\uparrow\rangle$ population into other (meta)stable states that are invisible for subsequent pulses on the selected transition. Alternatively, one may change the frequency for each of the excitation pulses, such that each pulse couples the ground state to a different excited electronic state. For ${ }^{40} \mathrm{Ca}^{+}$ions, there are six levels in the $S_{5 / 2}$ state manifold, and four in the $S_{3 / 2}$ manifold. Thus, the present scheme may be used for spectroscopic protocols of up to six or even ten pulses, depending on the available laser system (the pulse sequences discussed in our paper consist of two to four pulses). The combination of sideband and carrier pulses with phases $\phi$ and $\pi / 2$, respectively, then effectively yields the state (after discarding the excited-state population by either one of the two alternatives above)

$$
\left|\Psi_{f}\right\rangle \simeq|\downarrow\rangle \otimes\left[\cos \left(\frac{\Omega_{n} t_{1}}{2}\right) \cos \left(\frac{\Omega t_{2}}{2}\right)|n\rangle-e^{i \phi} \sin \left(\frac{\Omega_{n} t_{1}}{2}\right) \sin \left(\frac{\Omega t_{2}}{2}\right)|n+1\rangle\right],
$$

which produces the desired coherence with phase signature $\phi$. The above expression corrects Eqs. (30) and (31), and the discussions of the incoherent pumping process become redundant. Apart from that, the remaining text also applies for the corrected result with $\rho_{f}=\left|\Psi_{f}\right\rangle\left\langle\Psi_{f}\right|$. The results of the paper are not affected.

We further correct some typographical errors:

(1) On page 2, in the paragraph preceding Eq. (3), the Green's functions should read $\mathcal{G}\left(\tau_{b}, \tau_{a}\right)=\mathcal{T} \exp \left[\int_{\tau_{a}}^{\tau_{b}} d t \mathcal{L}(t)\right]$ and $\mathcal{G}\left(\tau_{b}-\tau_{a}\right)=\exp \left[\mathcal{L}\left(\tau_{b}-\tau_{a}\right)\right]$.

(2) There is a typesetting error in Eq. (9), which instead should read

$$
S_{i_{1}, i_{2} ; j}^{(L l)}\left(t_{1}, t_{2}\right)=\operatorname{Tr}\left\{O_{j} \mathcal{G}\left(t_{2}\right) \mathcal{A}_{i_{2}}^{(l)} \mathcal{G}\left(t_{1}\right) \mathcal{A}_{i_{1}}^{(L)} \rho(0)\right\} .
$$

(3) Below Eq. (17), in the third line, it should read $\beta_{0}=v_{z}^{2} / v_{x}^{2}$.

(4) For consistency with Fig. 4(a), the notation in Eq. (25) should be

$$
v_{1}-v_{2}=v_{x} .
$$

(5) In writing expression (43) we assumed that the frequency $v_{x}$ is normalized to one. The general expression reads

$$
H=\left(\begin{array}{ccc}
v_{x}-t_{12}-t_{13} & t_{12} & t_{13} \\
t_{12} & v_{x}-2 t_{12} & t_{12} \\
t_{13} & t_{12} & v_{x}-t_{12}-t_{13}
\end{array}\right),
$$

which yields the single exciton energies $e_{1}=v_{x}-3 t_{12}, e_{2}=v_{x}-t_{12}-2 t_{13}$, and $e_{3}=v_{x}$. 